\title{
Genetically-modified stem cells in treatment of human diseases: Tissue kallikrein (KLK1)-based targeted therapy (Review)
}

\author{
MARINA DEVETZI $^{1}$, MARIA GOULIELMAKI ${ }^{1}$, NICOLAS KHOURY ${ }^{1}$, DEMETRIOS A. SPANDIDOS ${ }^{2}$, \\ GEORGIA SOTIROPOULOU ${ }^{3}$, IOANNIS CHRISTODOULOU ${ }^{1}$ and VASSILIS ZOUMPOURLIS ${ }^{1}$ \\ ${ }^{1}$ Biomedical Applications Unit, Institute of Biology, Medicinal Chemistry and Biotechnology, \\ National Hellenic Research Foundation, 11635 Athens; ${ }^{2}$ Laboratory of Clinical Virology, Medical School, \\ University of Crete, 71003 Heraklion; ${ }^{3}$ Department of Pharmacy, University of Patras, Rion, 26500 Patras, Greece
}

Received November 2, 2017; Accepted January 2, 2018

DOI: $10.3892 /$ ijmm.2018.3361

\begin{abstract}
The tissue kallikrein-kinin system (KKS) is an endogenous multiprotein metabolic cascade which is implicated in the homeostasis of the cardiovascular, renal and central nervous system. Human tissue kallikrein (KLK1) is a serine protease, component of the KKS that has been demonstrated to exert pleiotropic beneficial effects in protection from tissue injury through its anti-inflammatory, anti-apoptotic, anti-fibrotic and anti-oxidative actions. Mesenchymal stem cells (MSCs) or endothelial progenitor cells (EPCs) constitute populations of well-characterized, readily obtainable multipotent cells with special immunomodulatory, migratory and paracrine properties rendering them appealing potential therapeutics in experimental animal models of various diseases. Genetic modification enhances their inherent properties. MSCs or EPCs are competent cellular vehicles for drug and/or gene delivery in the targeted treatment of diseases. $K L K 1$ gene delivery using adenoviral vectors or KLK1 protein infusion into injured tissues of animal models has provided particularly encouraging results in attenuating or reversing myocardial, renal and cerebrovascular ischemic phenotype and tissue damage, thus paving the way for the administration of genetically modified MSCs or EPCs with the human tissue $K L K 1$ gene. Engraftment of KLK1-modified MSCs and/or $K L K 1$-modified EPCs resulted in advanced beneficial outcome regarding heart and kidney protection and recovery from ischemic insults. Collectively, findings from pre-clinical studies raise the possibility that tissue KLK1 may be a novel future therapeutic target in the treatment of a wide range of cardiovascular, cerebrovascular and renal disorders.
\end{abstract}

Correspondence to: Dr Vassilis Zoumpourlis, Biomedical Applications Unit, Institute of Biology, Medicinal Chemistry and Biotechnology, National Hellenic Research Foundation, 48 Vasileos Constantinou Avenue, 11635 Athens, Greece

E-mail:vzub@eie.gr

Key words: tissue kallikrein (KLK1), kallikrein-kinin system, stem cells, genetic modification, targeted therapy

\section{Contents}

1. Introduction

2. Stem cell properties

3. Therapeutic implications of naïve stem cells

4. Genetically-modified stem cells in treatment of human diseases

5. Tissue KLK1-modified stem cells in cardiac and vascular diseases

6. Tissue $K L K 1$-modified stem cells in renal diseases

7. Tissue $K L K 1$-gene delivery in neurological diseases

8. Conclusion

\section{Introduction}

The tissue kallikrein-kinin system (KKS) is an endogenous multiprotein metabolic cascade which is implicated in a plethora of biological processes such as inflammation, vasodilation, blood coagulation, fibrinolysis, blood pressure control, vascular permeability, cardioprotection, smooth muscle contraction, electrolyte balance and pain induction. Activation of KKS leads to synthesis of the vasoactive peptides kinins by enzymatic hydrolysis of precursor kininogens including high molecular weight kininogen (HMWK) and low molecular weight kininogen (LMWK) $(1,2)$. Kininogens are physiological substrates for proteolytic cleavage by a family of serine proteases consisting of kallikreins (KLKs) originating from plasma (pKLK) and tissue (tKLK) (3). Human plasma kallikrein (KLKB1) is a serine protease synthesized predominantly in the liver that possesses a high affinity binding site for HMWK, which is cleaved to produce the nonapeptide bradykinin. Human tissue kallikrein (KLK1) is a serine protease of the S1 serine protease superfamily which cleaves LMWK to produce the decapeptide Lys-bradykinin (kallidin) that is further processed to bradykinin by a second aminopeptidase cleavage. Bradykinin is the basic vasoactive peptide of the KKS involved in the regulation of blood pressure as well as flow. Bradykinin-related peptides bind to B1 and B2 bradykinin receptors in order to activate a number of downstream targets such as nitric oxide (NO), cGMP, prostacyclin and cAMP, which in turn induce numerous biological processes implicated in angiogenesis 
by stimulation of vascular endothelial growth factor (VEGF) formation through binding to B2 receptor, increase of vascular permeability, vasodilation, smooth muscle contraction/relaxation, inflammation and pain (4-8). In addition, KLK1 protease showing both trypsin- and chymotrypsin-like specificity, appears to have many physiological protein substrates including pro-insulin, pro-renin, low-density lipoprotein, and the matrix metalloproteinases (MMPs) pro-gelatinase (MMP-2) and pro-collagenase (MMP-9) $(9,10)$.

Human mesenchymal stem cells (MSCs) are multipotent fibroblast-like somatic cells with the ability to self-renew, proliferate and differentiate in order to give rise to tissue- or organ-specific cells of the mesodermal lineage (e.g., osteoblasts, chondrocytes, adipocytes, stroma cells, skeletal myoblasts and endothelial cells). MSCs are a heterogeneous subset of stromal stem cells that can be isolated from many different adult or fetal tissue sources including bone marrow, adipose tissue, umbilical cord blood, amniotic fluid, synovial fluid, peripheral blood, dermis, liver, skin and skeletal muscle (11-14). As determined by the International Society for Cellular Therapy (ISCT), human MSCs must meet the following minimum criteria: adherence to tissue culture plastic under standard culture conditions, expression of cell surface molecules CD105, CD73 and CD90 and lack of expression of CD45, CD34, CD14 or CD11b, CD79 $\alpha$ or CD19 and HLA class II and capability of differentiating into adipocytes, osteocytes and chondrocytes under standard experimental conditions in vitro (15).

Another stem cell population which has been proposed as remarkable candidate for stem cell therapy is human endothelial progenitor cells (EPCs). EPCs are precursor cells that have the potential to differentiate into mature endothelial cells and can be isolated from bone marrow aspirate or peripheral blood of adult organisms. EPCs participate in the processes of postnatal formation of new blood vessels and recovery of damaged tissues by incorporating into the vasculature and by secreting vasculogenic cytokines and proangiogenic factors such as VEGF, angiopoietin-1 (Ang1), hepatocyte growth factor (HGF), platelet-derived growth factor (PDGF), monocyte chemoattractant protein-1 (MCP-1), and macrophage inflammatory protein-1 (MIP-1) (16-20). Vasculogenic cytokines recruit EPCs to the process of healing in response to hypoxia or ischemia, whereas proangiogenic cytokines regulate EPC mobilization, homing, proliferation, and differentiation. The angiogenic potency of EPCs is also demonstrated through their tube formation capacity in in vitro assays or when injected to murine models. EPCs also contribute to neovascularization and tissue repair of musculoskeletal and neural tissue including the bone and spinal cord. Transplantation of EPCs has been used to treat ischemic diseases in animal models and clinical trials (20-22).

\section{Stem cell properties}

Key properties of human MSCs are their immunomodulatory capability and their marked propensity to migrate towards sites of injury or inflammation (tropism). Due to these special characteristics, MSCs have been highlighted as promising tools for clinical use in regenerative medicine as well as targeted cell therapy of various diseases including cardiovascular, cerebrovascular, renal, autoimmune disorders and cancer $(13,23,24)$.
MSCs of various origin can be readily extracted from adult tissues and expanded in vitro without the loss of their potential for clinical applications or differentiation into multiple cell lineages $(14,25)$.

One of the most intriguing features of MSCs is that they can interact with cells of both the innate and adaptive immune systems and modulate their effector functions by secreting several cytokines. Interleukins 10 (IL-10) and 8 (IL-8) and transforming growth factor- $\beta$ (TGF- $\beta$ ) produced by MSCs lead to repression of immune responses and promotion of tissue healing. MSC-mediated immunomodulation results in MSC escape from host immunological recognition and rejection in allogeneic injection due to lack of major histocompatibility complex MHC-II and only minimal MHC-I protein expression $(13,24,26)$.

The other crucial feature of MSCs is that they can physiologically perfuse into the peripheral blood and migrate to injured or inflamed tissues (tropism), where they can inhibit the release of pro-inflammatory cytokines and promote the survival of damaged cells $(24,27)$. MSC tropism is mediated through paracrine signaling between the site of injury and corresponding receptor expression on MSCs (23). For example, stromal cell-derived factor-1 (SDF-1) is one of the main chemokines mediating the mobilization and homing of stem cells to damaged tissues and was found to improve repairing efficiency (28). These unique properties render MSCs ideal vehicles for cellular gene transfer.

Interestingly, there is an MSC population that has been particularly highlighted for its unique characteristics: The MSCs derived from the Wharton's Jelly (WJ-MSCs) - an anatomic region within the umbilical cord. WJ-MSCs are primitive cells categorized somewhere between embryonic stem cells (ESCs) and adult stem cells. Due to their immunogenic and functional superiority to other MSCs, a special mention of WJ-MSCs should be made. Similar to ESCs and unlike adult MSCs, they are consistently positive for pluripotency and self-renewal markers (29). Importantly, they are safer to use since they do not form teratomas in vivo (in contrast to ESCs) and sustain high proliferation rates for extended periods in culture with no signs of transformation, in contrast to adult MSCs that have been linked to transformation events as a result of replicative senescence (30). The most remarkable feature of WJ-MSCs is their hypo-immunogenic profile (a key requirement for allogeneic transplantation) and their capacity for immunomodulation (31). WJ-MSCs are capable of evading immune recognition due to their lack of co-stimulatory molecule expression, which is normally implicated in activation of $\mathrm{T}$ and $\mathrm{B}$ cell responses and they can also suppress allogeneically stimulated $\mathrm{T}$ cells to a greater extent than adult MSCs (32).

\section{Therapeutic implications of naïve stem cells}

Clinical trials using human MSCs of various origin as well as EPCs are currently underway to treat cardiovascular, cerebrovascular, renal, intestinal and autoimmune diseases.

Implications in cardiovascular disorders. Accumulating evidence from a variety of animal models of acute myocardial infarction (MI) injected or transplanted with MSCs, has 
demonstrated that MSCs constitute promising therapeutic tools for repairing and regenerating cardiac cells, interrupting the progress of left ventricular remodeling following acute MI and restoring heart structure and function by reducing infarct size and enhancing angiogenesis and arteriogenesis in the ischemic tissue (11,33-39). Their effects are attributed to their special properties of homing to injured tissues, self-proliferating and differentiating into cardiomyocytes in the damaged area. Indeed, MSCs are able to differentiate into cells that exhibit cardiomyocyte features in vitro, however, the proportion of MSCs that differentiate into cardiomyocytes in vivo and those that actually survive for a long period is very small $(40,41)$. As known, ischemia induces the production of reactive oxygen species (ROS) and a number of inflammatory molecules, such as tumor necrosis factor (TNF)- $\alpha$, intercellular adhesion molecule-1 (ICAM-1) and MCP-1 (42). After transplantation into the body, MSCs exhibit paracrine activity by secreting various cytokines including growth factors (e.g., VEGF) that produce anti-inflammatory as well as reparative effects. These molecules can decrease gene expression of inflammatory agents such as TNF- $\alpha$ and IL- $1 \beta$ and IL-6 and they can also promote survival, growth, or differentiation of other cells in the area of the MI, and this is considered the major contribution of MSCs in treatment efficacy $(37,41,43,44)$. The functionally superior WJ-MSCs transplanted by direct injection into the infarcted area of myocardium could survive and differentiate into cardiomyocytes and endothelial cells and also promoted recruitment and differentiation of cardiac stem cells in a porcine model. In addition, WJ-MSC transplantation was shown to reduce apoptosis and fibrosis, enhance viable myocardium, and improve ventricular remodeling and function (45). Scheduled and ongoing clinical trials test the efficacy and safety of these cells in patients with MI (e.g., clinicaltrials.gov; NCT01291329).

Implications in neurological disorders. MSCs have been reported as having significant neural differentiation potential in culture and being neurogenic after transplantation in rodent models, therefore they have gained interest in their potential usefulness in cell-based therapy strategies for neurodegenerative diseases and traumatic injuries of the nervous system $(11,46,47)$. Indeed, prolonged cultured bone marrow-derived MSCs can differentiate into neuron-like cells (48). Transplantation of bone marrow-derived MSCs to animal models of neurodegenerative disorders including Parkinson's disease and ischemic brain injury has been reported to ameliorate functional deficits $(49,50)$. The main challenge to stem cell therapy of central nervous system (CNS) diseases is getting MSCs into the CNS through the blood-brain barrier (51). When transplanted into the brain, MSCs produce neurotrophic and growth factors that protect and induce regeneration of damaged tissue. It has been shown that MSCs can differentiate into neurons and glial cells. Additionally, transplantation of MSCs enables the formation of new blood vessels, thereby increasing blood flow in the ischemic region. It has been shown that intravenous injection of umbilical cord blood-derived MSCs to transgenic mice with Alzheimer's disease results in a decline of cerebral amyloid $\beta$ $(\mathrm{A} \beta)$ peptide and an increase of this peptide in blood plasma due to its excretion from the brain through the blood-brain barrier, as well as a reduction of pro-inflammatory responses in the brain and periphery (52-55). Moreover, WJ-MSCs have been used for induction of neurons and glial cells (56) and they have been shown to promote functional and morphologic recovery of peripheral nerves after axonotmesis and neurotmesis injuries in a rat model (57). WJ-MSCs effectivity in patients with chronic traumatic spinal cord injury is under clinical trial (clinicaltrials.gov; NCT03003364).

Implications in renal disorders. Mounting evidence from ongoing or completed clinical trials indicates that MSC therapy is feasible, safe, well tolerated, and can effectively improve renal pathologies including acute kidney injury (AKI) and chronic kidney disease (CKD), diabetic nephropathy, focal segmental glomerulosclerosis (FSGS), systemic lupus erythematous (SLE), and kidney transplantation. Since regenerative capability of renal cells in humans is very limited, damage in these cells usually lead to devastating diseases. Numerous preclinical studies in various murine models have paved the way for novel therapeutic strategies with the use of MSCs and/or EPCs in a wide range of renal disorders both acute and chronic (58-60).

MSCs have a renoprotective and regenerative action on injured kidney tissues via paracrine mechanisms: anti-fibrotic, anti-apoptotic, pro-angiogenic, proliferative, differentiative, antioxidative, immunosuppressive and immunomodulatory. More specifically, paracrine release of extracellular vesicles including exosomes that contain genetic and protein material by MSCs has been proposed to exert trophic and reparative effects, which can activate mechanisms to ameliorate renal injury $(21,60)$. It has been shown that implantation of bone marrow-derived MSCs after ischemia/reperfusion (I/R)-induced acute renal failure promotes restoring of renal function and morphology, thereby implicating the great therapeutic potential of MSCs in healing damaged kidneys (61-63). Administration of MSCs has demonstrated significant reduction of intrarenal inflammatory infiltrate, decreased fibrosis, and glomerulosclerosis in animal models of CKD (59). Moreover, in animal models of diabetic nephropathy, MSCs reduced glomerulosclerosis and oxidative stress (64-66). Intrarenal delivery of MSCs and EPCs in a porcine model of renovascular hypertension resulted in decreased myocardial injury induced by renovascular hypertension as well as decreased renal injury $(67,68)$. In addition, the identification and characterization of adult renal progenitor cells from rodents as well as humans has provided further insights concerning stem cell regenerative potential in renal tubular injuries $(58,69,70)$. A meta-analysis of studies in animal models by Papazova et al demonstrated that cell-based therapy reduced development and progression of CKD by decreasing urinary protein and urea associated with glomerulosclerosis and interstitial fibrosis (71). Although MSC delivery in in vivo models of FSGS has been scarcely studied, results were promising as MSCs were shown to stabilize and attenuate the progression of FSGS $(72,73)$. Studies have shown that allogeneic bone marrow or umbilical cord-derived MSC transplantation results in amelioration of disease and could reverse multiorgan dysfunction in SLE (74,75). Treatment with MSCs exhibiting anti-inflammatory and immunomodulatory properties had either beneficial or no adverse effect on autoimmune 
lupus nephritis (the major clinical manifestation of SLE) as well as inflammatory bowel disease (IBD), but more research as to whether MSCs could actually benefit these patients is still underway (76,77). Furthermore, WJ-MSCs were shown to improve renal function in a xenogeneic mouse model of acute renal injury by increasing proliferation and decreasing apoptosis of renal tubular cells, a function mediated through the mitochondrial pathway, and through the increase of Akt phosphorylation (78). WJ-MSCs are being clinically tested in patients with diabetic nephropathy (clinicaltrials.gov; NCT03288571).

EPCs are reduced in number and impaired in angiogenic function in patients with atherosclerosis, cardiovascular and chronic renal diseases. Preclinical studies have shown that EPCs are mobilized from the bone marrow to peripheral blood in response to VEGF or other chemotactic molecules, home in at injured or inflamed tissues and differentiate into vessel-forming endothelial cells and/or regulate pre-existing endothelial cells (19,79-81). Moreover, EPCs attenuate vascular inflammation and improve left ventricular function both in vitro and in vivo, thereby being regarded as promising tools for post-MI therapy. Indeed, post-MI implantation of EPCs into ischemic myocardium of animal models can home to sites of injury and enhance recovery. The number and function of circulating EPCs has been inversely correlated with cardiovascular disease risk as a potential biomarker for patients with hypertension and coronary artery disease (18,80,82-86). Furthermore, EPCs have been proposed as useful tools in cell-based treatment of renal and ischemic cerebrovascular diseases. Studies have shown that mobilization of EPCs contributes to endothelial repair in the kidney immediately after I/R $(87,88)$. EPCs have been also shown to decrease neuronal apoptosis and promote the proliferation and migration of neural stem cells by repairing vascular endothelial cells and inducing neo-vascularization in animal models of cerebral ischemia. Growth factor (e.g., VEGF) secretion by EPCs contributes to post-stroke angiogenesis and neurogenesis, thereby reconstructing the functions and structures of vascular and neural networks $(22,89,90)$.

Stem cell therapy using naïve MSCs and/or EPCs for tissue regeneration confronts many challenges regarding stem cell viability, vitality and functionality. After extensive debate on these issues, research advances could finally provide safer applicable solutions.

\section{Genetically-modified stem cells in treatment of human diseases}

Genetic modification of human stem or progenitor cells (e.g., MSCs and/or EPCs) for targeted delivery of specific therapeutic agents or genes has been proven to be a very significant advancement in regenerative medicine, since it can improve viability, proliferative capability and metabolic features of these cells which are sensitive to the hypoxic and inflammatory environment in ischemic tissue. For example, MSCs overexpressing the anti-apoptotic gene Aktl (Akt-MSCs) were shown to be more resistant to apoptosis in vitro and in vivo (91). Moreover, the efficacy of MSCs for clinical use can be optimized by pre-treatment with drugs, cytokines, and growth factors $(92,93)$.
MSCs can be genetically modified by viral and non-viral methods. Non-viral physical and chemical methods of gene transfer are able to deliver larger transgenes than viral methods, but their main drawback is the low transfection efficiency and transient gene expression (94). MSCs can be efficiently transduced with viral vectors such as lentiviruses, retroviruses, baculoviruses and adenoviruses without affecting their stem cell properties. Viruses can be useful as delivery vectors after being considerably modified in order to become replication incompetent with attenuated cytopathic effects and immunogenicity. Viral vectors are particularly appealing because they can enable high transduction efficiency and, depending on the type of virus used, can deliver long-term stable transgene expression. The safety of cell-based therapy with the use of viral vectors is a crucial issue that has not been resolved yet, but advances in vector design have helped towards this direction $(23,95)$. For example, MSCs genetically modified to express VEGF have been shown to enhance the cardioprotective effects of MSCs followed by angiogenesis effects for the treatment of acute MI, whereas Akt gene- or heme oxygenase-1 (HO-1) gene-modified MSCs have been shown to dramatically improve ischemic cardiac function and MSC viability and prevent ventricular remodeling and apoptosis of cardiomyocytes and endothelial cells, thus restoring the function of infarcted hearts $(91,96-98)$. In addition, MSCs genetically modified with HGF or VEGF ameliorated acute renal damage, inflammation and apoptosis $(99,100)$.

Genes such as tissue $K L K 1$ that have been shown to inhibit inflammation, apoptosis, fibrosis and ROS formation, would be a choice for the genetic modification of MSCs and/or EPCs that are intended against organ or tissue injury in human diseases. Indeed, tissue $K L K 1$-modified MSCs (KLK1-MSCs) have been reported to play a protective role in cardiovascular, cerebrovascular and renal disorders in vivo as well as in vitro (63). In this review we discuss the advances in the use of $K L K 1$-MSCs and/or KLK1-EPCs in cell-based therapy of human diseases.

\section{Tissue $K L K 1$-modified stem cells in cardiac and vascular diseases}

The KKS through its components is a crucial regulator of homeostasis of the cardiovascular system throughout the life of an individual and has been implicated in blood pressure regulation (vasodilation) as well as pathogenesis of hypertension $(6,101)$. Since the discovery of tissue KLK1 localization in cardiac and vascular tissues (102-104), multiple experimental studies have investigated its role and potential therapeutic application both in vitro and in vivo. Tissue KLK1 has been demonstrated to exert pleiotropic beneficial effects in cardiovascular system function by reducing hypertension, attenuating cardiac inflammation and myocardial fibrosis, increasing NO formation, restoring coronary blood flow, decreasing infarct size, promoting neo-vascularization and capillary density and preventing restenosis after acute MI through the VEGF and kinin B2 receptor-Akt-glycogen synthase kinase (GSK)-3 $\beta$ signaling pathways (105-110). Many of these beneficial effects of KLK1 are mediated by the activation of NO signaling pathways, which are responsible for a decrease in oxidative stress in animal models. In vivo studies with gene delivery using adenoviral vectors that contained the human $K L K 1$ gene or 
with KLK1 protein infusion have shown that KLK1 reduces cardiac inflammation, hypertrophy, fibrosis and apoptosis of cardiomyocytes in animal models of MI (105-108,111-113). In vitro studies on cardiomyocytes and endothelial cells showed that tissue KLK1 expression inhibited hypoxia-induced ROS formation as well as cardiomyocyte apoptosis via activation of Akt-mediated signaling cascades (Akt-GSK-3 $\beta$ and AktBad-14-3-3) (4,108,109,114). Moreover, KLK1 gene delivery increases the population of cardiac progenitor cells (CPCs) and promotes viability, increases the regional blood flow and neo-vascularization in the peri-infarct myocardium (115). Adenovirus-mediated $K L K 1$ gene delivery in rodent models was also found to induce endogenous angiogenesis in response to ischemia, and to reduce neointima formation in injured vessels or after balloon angioplasty via activation of Akt and NO-cGMP signaling pathways (116-119). These data suggest that $K L K 1$ gene therapy might be applicable to peripheral occlusive vascular diseases.

Engineered MSCs can be used as vehicles to deliver therapeutic agents such as tissue KLK1 to injured end-organs. It has been shown that MSCs transduced with adenovirus containing the human tissue $K L K 1$ gene (KLK1-MSCs) acquire improved properties that augment their protective role in cardiovascular diseases. KLK1-MSCs secrete KLK1 which may contribute to the reduction in myocardial fibrosis via proteolytic activation of pro-MMP-2 and -9. MMPs are known to degrade the physiological collagen scaffold of the myocardium and other extracellular matrix (ECM) proteins. KLK1-MSCs also express higher levels of VEGF and VEGF-R compared to unmodified MSCs $(39,107,120,121)$. The upregulation of VEGF and its receptor could partly account for KLK1-induced neo-vascularization in the infarct myocardium (107). The in vivo pro-angiogenic effects of KLK1-MSCs exhibiting augmented VEGF secretion were additionally confirmed in vitro in cultured endothelial cells wherein a significant increase of proliferation, migration and tube formation was observed (39). KLK1 can also inhibit collagen synthesis and promote collagen breakdown. Notably, administration of KLK1-MSCs to rats reduces cardiac collagen deposition and cardiomyocyte hypertrophy $(39,122)$. Moreover, KLK1-MSCs show reduced caspase-3 activity compared to controls and their administration to rats decreased myocardial apoptosis after MI. Cultured KLK1-MSCs are more resistant to hypoxia-induced apoptosis compared to control MSCs and this resistance possibly enables engraftment of KLK1-MSCs to the infarct area in larger amount than control MSCs. Administration of KLK1-MSCs to rats also resulted in significant decrease of inflammatory cell (neutrophil and monocyte/macrophage) accumulation in the myocardium and parallel downregulation of TNF- $\alpha$, ICAM-1 and MCP-1 after MI (39). It has also been suggested that KLK1-MSCs could provide significant cardioprotection possibly due to the ability of KLK1 to activate the kinin B2 receptor to either form kinins or not, thereby attenuating myocardial damage through Akt signaling and NO production $(39,106)$. Taken together, the aforementioned findings converge to the notion that MSCs modified with human tissue $K L K 1$ gene constitute appealing therapeutics with multifaceted potential in cell-based therapy of myocardial ischemia.

EPCs have also been transduced with adenovirus containing the human tissue $K L K 1$ gene and studied in vitro and in vivo for the effects of their implantation in animal models of ischemia. Yao et al reported that genetic modification of EPCs with the human $K L K 1$ gene induces Akt phosphorylation and VEGF expression in response to oxidative stress. KLK1-EPCs provided enhanced cardioprotection in rats by preventing cardiomyocyte apoptosis, reducing infarct size, restoring left ventricular function and increasing therapeutic angiogenesis and arteriogenesis after acute ischemia-induced MI $(85,123)$. The angiogenic activity of cultured KLK1-EPCs is promoted by increased expression of endothelial NO synthase (eNOS) and integrin $\alpha v \beta 3$ on the surface of EPCs (19). Furthermore, the increased $K L K 1$ expression levels lead to enhanced cell proliferation, adhesion, migration, invasion, and tube formation and decreased hypoxia-induced apoptosis in cultured $K L K 1$-EPCs $(19,85)$. Importantly, KLK1-EPCs exhibited significant retention and viability in ischemic heart which is an important factor for preservation of cardiac function. Fu et al also found that the administration of $K L K 1$-EPCs into the caudal vein of ischemic rats results in a more effective increase of muscular capillary density, blood flow and myofiber number in an induced hindlimb ischemia rat model in comparison to administration of unmodified or control EPCs (19).

\section{Tissue $K L K 1$-modified stem cells in renal diseases}

The renal KKS is involved in electrolyte and water homeostasis, blood pressure regulation and inflammation. Historically, tissue KLK1 was discovered in human urine at the beginning of the twentieth century as a substance exerting hypotensive action. KLK1 is localized in the collecting segment of the renal distal tubule and its release into the tubules can be induced by the electrolyte balance (low sodium levels, high potassium levels) and antidiuretic hormone $(114,124)$. KLK1 renal excretion in urine is decreased in hypertensive rodents and humans to an extent that is proportional to the severity of renal failure. This decrease might result from a decrease in kinin generation (e.g., bradykinin) in hypertensive conditions, since kininogen levels and kinin-forming factors are reduced in essential and malignant hypertension. It has been suggested that the role of renal bradykinin is to excrete the excess sodium. Therefore, decrease in renal bradykinin generation may lead to sodium accumulation in the body which in turn could result in the development of hypertension (124-126).

Several studies have shown that KLK1 improves renal function by increasing glomerular filtration rate and renal blood flow via its anti-inflammatory, anti-oxidative, anti-fibrotic and anti-apoptotic actions in animal models of renal injury. For example, $K L K 1$ gene delivery using an adenovirus vector or KLK1 protein infusion in hypertensive Dahl salt-sensitive rats has been shown to attenuate renal dysfunction, induce NO production and reverse the process of renal inflammation and fibrosis in bradykinin B2 receptor-mediated manner (127-131). Liu et al confirmed the previous findings by blocking endogenous KLK1 activity in a rat model of CKD which resulted in increased inflammatory cell (macrophages/monocytes) infiltration and myofibroblast and collagen deposition in kidneys (132). Specifically, endogenous KLK1 was shown to inhibit angiotensin II-induced ROS and superoxide formation as well as renal NADH oxidase activity through NO production in deoxycorticosterone acetate (DOCA)-salt 


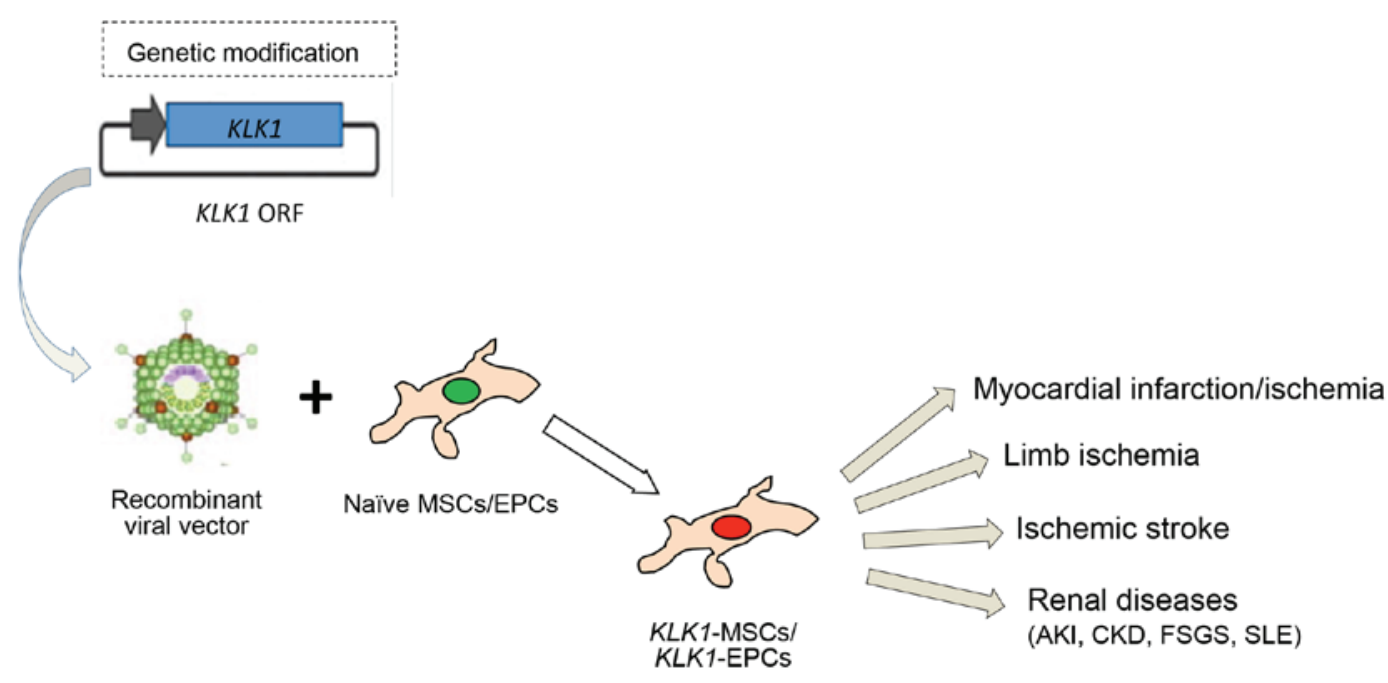

Figure 1. Genetic modification strategy for the insertion of $K L K 1$ gene into naïve stem cells and possible therapeutic applications. MSCs, mesenchymal stem cells; EPCs, endothelial progenitor cells; AKI, acute kidney injury; CKD, chronic kidney disease; FSGS, focal segmental glomerulosclerosis; SLE, systemic lupus erythematous.

hypertensive rats, which exhibit high renal KLK1 levels. Moreover, KLK1 significantly increased MMP-2 activity and inhibited synthesis of tissue inhibitor of MMP-2 (TIMP-2) as well as plasminogen activator inhibitor-1 (PAI-1), thereby promoting degradation of ECM protein components (e.g., collagen I and fibronectin) as demonstrated both in vivo and in cultured renal cells $(63,132,133)$. Most likely, KLK1 exerts its anti-fibrotic effect by increasing ECM degradation which leads to a decrease of mesenchymal fibroblast accumulation in the interstitium of the cortex and medulla. Moreover, KLK1 was demonstrated to decrease renal hypertrophy, namely kidney weight, glomerular size, and proliferation of epithelial tubular cells. KLK1 protein infusion in rats also promoted the recovery of gentamicin-induced nephrotoxicity by inhibiting apoptosis and caspase- 3 activity and increasing Akt phosphorylation in proximal tubular renal cells. Therefore, endogenous tissue KLK1 can attenuate and reverse renal injury by reducing oxidative stress, apoptosis, inflammation and fibrosis in vivo through activation of bradykinin B2 receptor (63,114,133-135). Overall, these studies outline the beneficial role of tissue KLK1 in the preservation of kidney structure and function by promoting tissue repair and regeneration in AKI and modulating the progression of CKD.

As already mentioned, $K L K 1$ modified MSCs secrete recombinant human KLK1 as well as increased VEGF levels into culture medium, and exhibit augmented survival under oxidative stress conditions compared to control MSCs. After systemic injection in rats, KLK1-MSCs migrated to the injured kidney and KLK1 overexpression was detected in rat glomeruli after I/R injury. KLKI-MSCs implantation in rat kidney provided advanced protection against ischemia-induced kidney injury by suppression of apoptosis and interstitial inflammatory cell accumulation. The engraftment of KLK1-MSCs reduced blood urea nitrogen, serum creatinine levels, and tubular injury. VEGF secretion from $K L K 1$-MSCs may be partly responsible for the improvement of renal injury, as well $(114,120)$. Moreover, the KKS has been shown to be involved in the acute manifestations of lupus nephritis which occurs when antibodies and complement components accumulate and cause inflammation of the kidney in SLE patients (136). KLK1, among other members of the KLK family, was found to exert a protective role against SLE and anti-glomerular basement membrane (anti-GBM) antibody-induced nephritis in rodents as well as humans (137). Li et al studied the effects of KLK1-MSC administration into murine injured kidneys and confirmed that KLK1 attenuated spontaneous lupus nephritis in mice (138). KLKl-MSCs displayed a remarkable protective effect against anti-GBM induced-nephritis and lupus nephritis compared to control MSCs by inhibiting oxidative stress, renal cell apoptosis and inflammatory cell infiltration into the kidneys of nephritic mice, in line with findings of Hagiwara et al (120). Taken together, the above presented data suggest that KLK1-modified MSCs have the potential to be used as therapeutic agents in a wide variety of renal diseases.

\section{Tissue KLK1-gene delivery in neurological diseases}

The KKS is capable of dilating cerebral arterial vessels partly because of the release of endothelium-derived relaxing factor $\mathrm{NO}$ which plays a complex role in cerebral ischemia. Ischemic conditions trigger an excessive activation of neuronal NO synthase (NOS), which results in production of NO that is toxic to surrounding neurons, but critical in maintaining cerebral blood flow and reducing infarct volume. The KKS through participation in NOS activation and following NO formation is implicated in endothelial cell function in the setting of ischemic stroke (139).

Despite the lack of studies using KLK1 modified MSCs in models of cerebrovascular or neurodegenerative diseases, we review current knowledge on the effects of adenovirus-mediated $K L K 1$ gene delivery in animal models of cerebral ischemia/ischemic stroke. Zhang et al have demonstrated that $K L K 1$ gene transfer attenuates the blood pressure rise and cerebral damage in hypertensive Dahl salt-sensitive rats leading to a decrease in the stroke-induced mortality rate (140). Subsequent studies have shown that intracerebroventricular injection of adenovirus carrying the $K L K 1$ gene prevents 
stroke-induced ischemic brain injury (cerebral infarction) by inhibiting neuronal and glial apoptosis and inflammation while promoting neurogenesis and angiogenesis in the ischemic brain of a rat model as well as in vitro $(139,141,142)$. Moreover, administration of tissue KLK1 can specifically stimulate the proliferation of murine neural stem cells only (no other neural cell types) independent of kinin formation, but without inducing their differentiation into neurons or glial cells (143). Tissue KLK1 administration can also suppress glutamate- or acidosis-mediated neurotoxicity in vitro and protect from hypoxia/reoxygenation-induced neuronal injury by promoting neuron viability at least partially through the KLK1-B2R-ERK1/2 signaling pathway (144). Collectively, these findings raise the possibility that tissue KLK1 may be a novel therapeutic target in the treatment of ischemic stroke-induced brain injuries paving the way for KLK1-MSC administration research in neurological diseases.

\section{Conclusion}

Numerous studies have outlined the pleiotropic beneficial effects of tissue KLK1 protease, component of the KKS, in the protection of cardiovascular, renal and central nervous systems from tissue injury. Genetic modification of stem cells or progenitor cells with $K L K 1$ gene enhances their viability and proliferative, migratory and functional properties, thus increasing their tissue healing effects in various human diseases (Fig. 1). Engraftment of KLK1-MSCs and/or KLK1-EPCs into animal models provided advanced protection against vascular and organ damage. Aforementioned findings reveal the KLK1 relevance to human diseases and pave the way for further research on the potential therapeutic perspectives of $K L K 1$-MSCs and/or $K L K 1$-EPCs that could lead to the translation of preclinical studies into effective and safe targeted therapies for cardiovascular, cerebrovascular and renal diseases.

\section{Acknowledgements}

The present study was supported by IKY Fellowships of Excellence for Postgraduate Studies in Greece - SIEMENS Program (2016-2017).

\section{References}

1. Moreau ME, Garbacki N, Molinaro G, Brown NJ, Marceau F and Adam A: The kallikrein-kinin system: Current and future pharmacological targets. J Pharmacol Sci 99: 6-38, 2005.

2. Kashuba E, Bailey J, Allsup D and Cawkwell L: The kininkallikrein system: Physiological roles, pathophysiology and its relationship to cancer biomarkers. Biomarkers 18: 279-296, 2013.

3. Bourdet B, Pécher C, Minville V, Jaafar A, Allard J, Blaes N, Girolami JP and Tack I: Distribution and expression of B2-kinin receptor on human leukocyte subsets in young adults and elderly using flow cytometry. Neuropeptides 44: 155-161, 2010.

4. Chao J, Bledsoe G, Yin H and Chao L: The tissue kallikrein-kinin system protects against cardiovascular and renal diseases and ischemic stroke independently of blood pressure reduction. Biol Chem 387: 665-675, 2006.

5. Emami N and Diamandis EP: New insights into the functional mechanisms and clinical applications of the kallikrein-related peptidase family. Mol Oncol 1: 269-287, 2007.

6. Bryant JW and Shariat-Madar Z: Human plasma kallikrein-kinin system: Physiological and biochemical parameters. Cardiovasc Hematol Agents Med Chem 7: 234-250, 2009.
7. Hillmeister P and Persson PB: The kallikrein-kinin system. Acta Physiol (Oxf) 206: 215-219, 2012.

8. Björkqvist J, Jämsä A and Renné T: Plasma kallikrein: The bradykinin-producing enzyme. Thromb Haemost 110: 399-407, 2013.

9. Borgoño CA and Diamandis EP: The emerging roles of human tissue kallikreins in cancer. Nat Rev Cancer 4: 876-890, 2004.

10. Sotiropoulou G, Pampalakis G and Diamandis EP: Functional roles of human kallikrein-related peptidases. J Biol Chem 284: 32989-32994, 2009.

11. Lee KD: Applications of mesenchymal stem cells: An updated review. Chang Gung Med J 31: 228-236, 2008.

12. Christodoulou I, Kolisis FN, Papaevangeliou D and Zoumpourlis V: Comparative evaluation of human mesenchymal stem cells of fetal (Wharton's jelly) and adult (adipose tissue) origin during prolonged in vitro expansion: Considerations for cytotherapy. Stem Cells Int 2013: 246134, 2013.

13. Shafei AE, Ali MA, Ghanem HG, Shehata AI, Abdelgawad AA, Handal HR, Talaat KA, Ashaal AE and El-Shal AS: Mesenchymal stem cells therapy: A promising cell based therapy for treatment of myocardial infraction. J Gene Med 19: e2995, 2017.

14. Rhee KJ, Lee JI and Eom YW: Mesenchymal stem cell-mediated effects of tumor support or suppression. Int J Mol Sci 16: 30015-30033, 2015.

15. Dominici M, Le Blanc K, Mueller I, Slaper-Cortenbach I, Marini F, Krause D, Deans R, Keating A, Prockop DJ and Horwitz E: Minimal criteria for defining multipotent mesenchymal stromal cells. The International Society for Cellular Therapy position statement. Cytotherapy 8: 315-317, 2006.

16. Rehman J, Li J, Orschell CM and March KL: Peripheral blood 'endothelial progenitor cells' are derived from monocyte/macrophages and secrete angiogenic growth factors. Circulation 107: 1164-1169, 2003

17. Hristov $M$ and Weber C: Endothelial progenitor cells: Characterization, pathophysiology, and possible clinical relevance. J Cell Mol Med 8: 498-508, 2004.

18. Kränkel N, Lüscher TF and Landmesser U: 'Endothelial progenitor cells' as a therapeutic strategy in cardiovascular disease. Curr Vasc Pharmacol 10: 107-124, 2012.

19. Fu SS, Li FJ, Wang YY, You AB, Qie YL, Meng X, Li JR, Li BC, Zhang Y and Da Li Q: Kallikrein gene-modified EPCs induce angiogenesis in rats with ischemic hindlimb and correlate with integrin $\alpha v \beta 3$ expression. PLoS One 8: e73035, 2013.

20. Kamei N, Atesok K and Ochi M: The use of endothelial progenitor cells for the regeneration of musculoskeletal and neural tissues. Stem Cells Int 2017: 1960804, 2017.

21. Hickson LJ, Eirin A and Lerman LO: Challenges and opportunities for stem cell therapy in patients with chronic kidney disease. Kidney Int 89: 767-778, 2016.

22. Liao S, Luo C, Cao B, Hu H, Wang S, Yue H, Chen L and Zhou Z: Endothelial progenitor cells for ischemic stroke: Update on basic research and application. Stem Cells Int 2017: 2193432, 2017.

23. Sage EK, Thakrar RM and Janes SM: Genetically modified mesenchymal stromal cells in cancer therapy. Cytotherapy 18: 1435-1445, 2016.

24. Uccelli A, Moretta L and Pistoia V: Mesenchymal stem cells in health and disease. Nat Rev Immunol 8: 726-736, 2008.

25. Mishra PJ, Mishra PJ, Glod JW and Banerjee D: Mesenchymal stem cells: Flip side of the coin. Cancer Res 69: 1255-1258, 2009.

26. YeZ, Wang Y, Xie HY and Zheng SS: Immunosuppressive effects of rat mesenchymal stem cells: Involvement of CD $4{ }^{+} \mathrm{CD} 25^{+}$regulatory T cells. Hepatobiliary Pancreat Dis Int 7: 608-614, 2008.

27. Borlongan CV: Bone marrow stem cell mobilization in stroke: A 'bonehead' may be good after all! Leukemia 25: 1674-1686, 2011.

28. Kortesidis A, Zannettino A, Isenmann S, Shi S, Lapidot T and Gronthos S: Stromal-derived factor-1 promotes the growth, survival, and development of human bone marrow stromal stem cells. Blood 105: 3793-3801, 2005.

29. Fong CY, Richards M, Manasi N, Biswas A and Bongso A: Comparative growth behaviour and characterization of stem cells from human Wharton's jelly. Reprod Biomed Online 15: 708-718, 2007.

30. Fong CY, Chak LL, Biswas A, Tan JH, Gauthaman K, Chan WK and Bongso A: Human Wharton's jelly stem cells have unique transcriptome profiles compared to human embryonic stem cells and other mesenchymal stem cells. Stem Cell Rev 7: 1-16, 2011.

31. Weiss ML, Anderson C, Medicetty S, Seshareddy KB, Weiss RJ, VanderWerff I, Troyer D and McIntosh KR: Immune properties of human umbilical cord Wharton's jelly-derived cells. Stem Cells 26: 2865-2874, 2008. 
32. Prasanna SJ and Jahnavi VS: Wharton's jelly mesenchymal stem cells as off-the-shelf cellular therapeutics: A closer look into their regenerative and immunomodulatory properties. Open Tissue Eng Regen Med J 4: 28-38, 2011.

33. Yoon J, Min BG, Kim Y-H, Shim WJ, Ro YM and Lim D-S: Differentiation, engraftment and functional effects of pre-treated mesenchymal stem cells in a rat myocardial infarct model. Acta Cardiol 60: 277-284, 2005.

34. Tang J, Xie Q, Pan G, Wang J and Wang M: Mesenchymal stem cells participate in angiogenesis and improve heart function in rat model of myocardial ischemia with reperfusion. Eur J Cardiothorac Surg 30: 353-361, 2006.

35. Wolf D, Reinhard A, Krause U, Seckinger A, Katus HA, Kuecherer $\mathrm{H}$ and Hansen A: Stem cell therapy improves myocardial perfusion and cardiac synchronicity: New application for echocardiography. J Am Soc Echocardiogr 20: 512-520, 2007

36. Yang J, Zhou W, Zheng W, Ma Y, Lin L, Tang T, Liu J, Yu J, Zhou X and Hu J: Effects of myocardial transplantation of marrow mesenchymal stem cells transfected with vascular endothelial growth factor for the improvement of heart function and angiogenesis after myocardial infarction. Cardiology 107: 17-29, 2007.

37. Guo J, Lin GS, Bao CY, Hu ZM and Hu MY: Anti-inflammation role for mesenchymal stem cells transplantation in myocardial infarction. Inflammation 30: 97-104, 2007.

38. Xu X, Xu Z, Xu Y and Cui G: Effects of mesenchymal stem cell transplantation on extracellular matrix after myocardial infarction in rats. Coron Artery Dis 16: 245-255, 2005.

39. Gao L, Bledsoe G, Yin H, Shen B, Chao L and Chao J: Tissue kallikrein-modified mesenchymal stem cells provide enhanced protection against ischemic cardiac injury after myocardial infarction. Circ J 77: 2134-2144, 2013.

40. Amado LC, Saliaris AP, Schuleri KH, St John M, Xie JS Cattaneo S, Durand DJ, Fitton T, Kuang JQ, Stewart G, et al: Cardiac repair with intramyocardial injection of allogeneic mesenchymal stem cells after myocardial infarction. Proc Natl Acad Sci USA 102: 11474-11479, 2005.

41. Goradel NH, Hoor FG, Negahdari B, Malekshahi ZV, Hashemzehi M, Masoudifar A and Mirzaei H: Stem cell therapy: A new therapeutic option for cardiovascular diseases. J Cell Biochem 119: 95-104, 2018

42. Chen XL, Zhang Q, Zhao R and Medford RM: Superoxide, $\mathrm{H}_{2} \mathrm{O}_{2}$, and iron are required for TNF-alpha-induced MCP-1 gene expression in endothelial cells: Role of Rac1 and NADPH oxidase. Am J Physiol Heart Circ Physiol 286: H1001-H1007, 2004.

43. Caplan AI and Dennis JE: Mesenchymal stem cells as trophic mediators. J Cell Biochem 98: 1076-1084, 2006.

44. Choi SH, Jung SY, Kwon SM and Baek SH: Perspectives on stem cell therapy for cardiac regeneration. Advances and challenges Circ J 76: 1307-1312, 2012.

45. Zhang W, Liu XC, Yang L, Zhu DL, Zhang YD, Chen Y and Zhang HY: Wharton's jelly-derived mesenchymal stem cells promote myocardial regeneration and cardiac repair after miniswine acute myocardial infarction. Coron Artery Dis 24: 549-558, 2013.

46. Deng J, Petersen BE, Steindler DA, Jorgensen ML and Laywell ED: Mesenchymal stem cells spontaneously express neural proteins in culture and are neurogenic after transplantation. Stem Cells 24: 1054-1064, 2006.

47. Tropel P, Platet N, Platel JC, Noël D, Albrieux M, Benabid AL and Berger F: Functional neuronal differentiation of bone marrow-derived mesenchymal stem cells. Stem Cells 24: 2868-2876, 2006.

48. Tseng PY, Chen CJ, Sheu CC, Yu CW and Huang YS: Spontaneous differentiation of adult rat marrow stromal cells in a long-term culture. J Vet Med Sci 69: 95-102, 2007.

49. Dezawa M, Hoshino M and Ide C: Treatment of neurodegenerative diseases using adult bone marrow stromal cell-derived neurons. Expert Opin Biol Ther 5: 427-435, 2005.

50. Kim EJ, Kim N and Cho SG: The potential use of mesenchymal stem cells in hematopoietic stem cell transplantation. Exp Mol Med 45: e2, 2013.

51. Aleynik A, Gernavage KM, Mourad YS, Sherman LS, Liu K, Gubenko YA and Rameshwar P: Stem cell delivery of therapies for brain disorders. Clin Transl Med 3: 24, 2014

52. Nikolic WV, Hou H, Town T, Zhu Y, Giunta B, Sanberg CD, Zeng J, Luo D, Ehrhart J, Mori T, et al: Peripherally administered human umbilical cord blood cells reduce parenchymal and vascular $\beta$-amyloid deposits in Alzheimer mice. Stem Cells Dev 17: 423-439, 2008
53. Tanna T and Sachan V: Mesenchymal stem cells: Potential in treatment of neurodegenerative diseases. Curr Stem Cell Res Ther 9: 513-521, 2014.

54. Galieva LR, Mukhamedshina YO, Arkhipova SS and Rizvanov AA Human umbilical cord blood cell transplantation in neuroregenerative strategies. Front Pharmacol 8: 628, 2017.

55. Lee NK, Na DL and Chang JW: Killing two birds with one stone: The multifunctional roles of mesenchymal stem cells in the treatment of neurodegenerative and muscle diseases. Histol Histopathol: Nov 30, 2017 (Epub ahead of print).

56. Gärtner A, Pereira T, Gomes R, Luís AL, França ML, Geuna S, Armada-da-Silva P and Maurício AC: Mesenchymal stem cells from extra-embryonic tissues for tissue engineering - regeneration of the peripheral nerve. In: Advances in Biomaterials Science and Biomedical Applications. Pignatello R (ed). InTech, 2013.

57. Ribeiro J, Gartner A, Pereira T, Gomes R, Lopes MA, Gonçalves C, Varejão A, Luís AL and Maurício AC: Perspectives of employing mesenchymal stem cells from the Wharton's jelly of the umbilical cord for peripheral nerve repair. Int Rev Neurobiol 108: 79-120, 2013.

58. Chambers BE and Wingert RA: Renal progenitors: Roles in kidney disease and regeneration. World J Stem Cells 8: 367-375, 2016.

59. Peired AJ, Sisti A and Romagnani P: Mesenchymal stem cell-based therapy for kidney disease: A review of clinical evidence. Stem Cells Int 2016: 4798639, 2016.

60. Aghajani Nargesi A, Lerman LO and Eirin A: Mesenchymal stem cell-derived extracellular vesicles for kidney repair: Current status and looming challenges. Stem Cell Res Ther 8: 273, 2017.

61. Tögel F, Hu Z, Weiss K, Isaac J, Lange C and Westenfelder C: Administered mesenchymal stem cells protect against ischemic acute renal failure through differentiation-independent mechanisms. Am J Physiol Renal Physiol 289: F31-F42, 2005.

62. Lange C, Tögel F, Ittrich H, Clayton F, Nolte-Ernsting C, Zander AR and Westenfelder C: Administered mesenchymal stem cells enhance recovery from ischemia/reperfusion-induced acute renal failure in rats. Kidney Int 68: 1613-1617, 2005.

63. Chao J, Bledsoe G and Chao L: Kallikrein-kinin in stem cell therapy. World J Stem Cells 6: 448-457, 2014

64. Ezquer F, Ezquer M, Simon V, Pardo F, Yañez A, Carpio D and Conget P: Endovenous administration of bone-marrow-derived multipotent mesenchymal stromal cells prevents renal failure in diabetic mice. Biol Blood Marrow Transplant 15: 1354-1365, 2009.

65. Fang Y, Tian X, Bai S, Fan J, Hou W, Tong H and Li D: Autologous transplantation of adipose-derived mesenchymal stem cells ameliorates streptozotocin-induced diabetic nephropathy in rats by inhibiting oxidative stress, pro-inflammatory cytokines and the p38 MAPK signaling pathway. Int J Mol Med 30: 85-92, 2012.

66. Castiglione RC, Maron-Gutierrez T, Barbosa CM, Ornellas FM, Barreira AL, Dibarros CB, Vasconcelos-dos-Santos A, Paredes BD, Pascarelli BM, Diaz BL, et al: Bone marrow-derived mononuclear cells promote improvement in glomerular function in rats with early diabetic nephropathy. Cell Physiol Biochem 32: 699-718, 2013.

67. Zhu XY, Urbieta-Caceres V, Krier JD, Textor SC, Lerman A and Lerman LO: Mesenchymal stem cells and endothelial progenitor cells decrease renal injury in experimental swine renal artery stenosis through different mechanisms. Stem Cells 31: 117-125, 2013.

68. Eirin A, Zhu XY, Krier JD, Tang H, Jordan KL, Grande JP, Lerman A, Textor SC and Lerman LO: Adipose tissue-derived mesenchymal stem cells improve revascularization outcomes to restore renal function in swine atherosclerotic renal artery stenosis. Stem Cells 30: 1030-1041, 2012.

69. Bussolati B, Bruno S, Grange C, Buttiglieri S, Deregibus MC, Cantino D and Camussi G: Isolation of renal progenitor cells from adult human kidney. Am J Pathol 166: 545-555, 2005.

70. Angelotti ML, Ronconi E, Ballerini L, Peired A, Mazzinghi B, Sagrinati C, Parente E, Gacci M, Carini M, Rotondi M, et al: Characterization of renal progenitors committed toward tubular lineage and their regenerative potential in renal tubular injury. Stem Cells 30: 1714-1725, 2012.

71. Papazova DA, Oosterhuis NR, Gremmels H, van Koppen A, Joles JA and Verhaar MC: Cell-based therapies for experimental chronic kidney disease: A systematic review and meta-analysis. Dis Model Mech 8: 281-293, 2015. 
72. Ma H, Wu Y, Xu Y, Sun L and Zhang X: Human umbilical mesenchymal stem cells attenuate the progression of focal segmental glomerulosclerosis. Am J Med Sci 346: 486-493, 2013.

73. Belingheri M, Lazzari L, Parazzi V, Groppali E, Biagi E, Gaipa G, Giordano R, Rastaldi MP, Croci D, Biondi A, et al: Allogeneic mesenchymal stem cell infusion for the stabilization of focal segmental glomerulosclerosis. Biologicals 41: 439-445, 2013.

74. Sun L, Akiyama K, Zhang H, Yamaza T, Hou Y, Zhao S, Xu T, Le A and Shi S: Mesenchymal stem cell transplantation reverses multiorgan dysfunction in systemic lupus erythematosus mice and humans. Stem Cells 27: 1421-1432, 2009.

75. Sun L, Wang D, Liang J, Zhang H, Feng X, Wang H, Hua B, Liu B, Ye S, Hu X, et al: Umbilical cord mesenchymal stem cell transplantation in severe and refractory systemic lupus erythematosus. Arthritis Rheum 62: 2467-2475, 2010.

76. Munir H and McGettrick HM: Mesenchymal stem cell therapy for autoimmune disease: Risks and rewards. Stem Cells Dev 24 2091-2100, 2015.

77.Flores AI,Gómez-Gómez GJ, Masedo-González Á and MartínezMontiel MP: Stem cell therapy in inflammatory bowel disease A promising therapeutic strategy? World J Stem Cells 7 : 343-351, 2015

78.Fang TC, Pang CY, Chiu SC, Ding DC and Tsai RK: Renoprotective effect of human umbilical cord-derived mesenchymal stem cells in immunodeficient mice suffering from acute kidney injury. PLoS One 7: e46504, 2012.

79. Werner $\mathrm{N}$ and Nickenig G: Endothelial progenitor cells in health and atherosclerotic disease. Ann Med 39: 82-90, 2007.

80.Jujo K, Ii M and Losordo DW: Endothelial progenitor cells in neovascularization of infarcted myocardium. J Mol Cell Cardiol 45: 530-544, 2008

81. Choi JH, Kim KL, Huh W, Kim B, Byun J, Suh W, Sung J, Jeon ES, Oh HY and Kim DK: Decreased number and impaired angiogenic function of endothelial progenitor cells in patients with chronic renal failure. Arterioscler Thromb Vasc Biol 24 1246-1252, 2004

82. Vasa M, Fichtlscherer S, Aicher A, Adler K, Urbich C, Martin H, Zeiher AM and Dimmeler S: Number and migratory activity of circulating endothelial progenitor cells inversely correlate with risk factors for coronary artery disease. Circ Res 89: E1-E7, 2001.

83. Schuh A, Liehn EA, Sasse A, Hristov M, Sobota R, Kelm M, Merx MW and Weber C: Transplantation of endothelial progenitor cells improves neovascularization and left ventricular function after myocardial infarction in a rat model. Basic Res Cardiol 103: 69-77, 2008.

84.Umemura T and Higashi Y: Endothelial progenitor cells: Therapeutic target for cardiovascular diseases. J Pharmacol Sci 108: 1-6, 2008

85. Yao Y, Sheng Z, Li Y, Yan F, Fu C, Li Y, Ma G, Liu N, Chao J and Chao L: Tissue kallikrein promotes cardiac neovascularization by enhancing endothelial progenitor cell functional capacity. Hum Gene Ther 23: 859-870, 2012

86. Simard T, Jung RG, Motazedian P, Di Santo P, Ramirez FD, Russo JJ, Labinaz A, Yousef A, Anantharam B, Pourdjabbar A and Hibbert B: Progenitor cells for arterial repair: Incremental advancements towards therapeutic reality. Stem Cells Int 2017: 8270498, 2017.

87. Kwon O, Miller S, Li N, Khan A, Kadry Z and Uemura T: Bone marrow-derived endothelial progenitor cells and endothelial cells may contribute to endothelial repair in the kidney immediately after ischemia-reperfusion. J Histochem Cytochem 58 687-694, 2010.

88. Patschan D, Krupincza K, Patschan S, Zhang Z, Hamby C and Goligorsky MS: Dynamics of mobilization and homing of endothelial progenitor cells after acute renal ischemia: Modulation by ischemic preconditioning. Am J Physiol Renal Physiol 291 F176-F185, 2006

89. Rosell A, Morancho A, Navarro-Sobrino M, Martínez-Saez E, Hernández-Guillamon $\mathrm{M}$, Lope-Piedrafita $\mathrm{S}$, Barceló V, Borrás F, Penalba A, García-Bonilla L and Montaner J: Factors secreted by endothelial progenitor cells enhance neurorepair responses after cerebral ischemia in mice. PLoS One 8: e73244, 2013

90.Li YF, Ren LN, Guo G, Cannella LA, Chernaya V, Samuel S, Liu SX, Wang H and Yang XF: Endothelial progenitor cells in ischemic stroke: An exploration from hypothesis to therapy. J Hematol Oncol 8: 33, 2015.
91. Mangi AA, Noiseux N, Kong D, He H, Rezvani M, Ingwall JS and Dzau VJ: Mesenchymal stem cells modified with Akt prevent remodeling and restore performance of infarcted hearts. Nat Med 9: 1195-1201, 2003

92.Dzau VJ, Gnecchi M and Pachori AS: Enhancing stem cell therapy through genetic modification. J Am Coll Cardiol 46: 1351-1353, 2005.

93. Mastri M, Lin H and Lee T: Enhancing the efficacy of mesenchymal stem cell therapy. World J Stem Cells 6: 82-93, 2014.

94.Park JS, Suryaprakash S, Lao YH and Leong KW: Engineering mesenchymal stem cells for regenerative medicine and drug delivery. Methods 84: 3-16, 2015.

95. Moradian Tehrani R, Verdi J, Noureddini M, Salehi R, Salarinia R, Mosalaei M, Simonian M, Alani B, Ghiasi MR, Jaafari MR, et al: Mesenchymal stem cells: A new platform for targeting suicide genes in cancer. J Cell Physiol: July 13, 2017 (Epub ahead of print).

96. Tang YL, Tang Y, Zhang YC, Qian K, Shen L and Phillips MI: Improved graft mesenchymal stem cell survival in ischemic heart with a hypoxia-regulated heme oxygenase-1 vector. J Am Coll Cardiol 46: 1339-1350, 2005.

97. Matsumoto R, Omura T, Yoshiyama M, Hayashi T, Inamoto S, Koh KR, Ohta K, Izumi Y, Nakamura Y, Akioka K, et al: Vascular endothelial growth factor-expressing mesenchymal stem cell transplantation for the treatment of acute myocardial infarction. Arterioscler Thromb Vasc Biol 25: 1168-1173, 2005.

98. Gnecchi M, He H, Melo LG, Noiseaux N, Morello F, de Boer RA, Zhang L, Pratt RE, Dzau VJ and Ingwall JS: Early beneficial effects of bone marrow-derived mesenchymal stem cells overexpressing Akt on cardiac metabolism after myocardial infarction. Stem Cells 27: 971-979, 2009.

99. Chen Y, Qian H, Zhu W, Zhang X, Yan Y, Ye S, Peng X, Li W and $\mathrm{Xu} \mathrm{W}$ : Hepatocyte growth factor modification promotes the amelioration effects of human umbilical cord mesenchymal stem cells on rat acute kidney injury. Stem Cells Dev 20: 103-113, 2011.

100. Yuan L, Wu MJ, Sun HY, Xiong J, Zhang Y, Liu CY, Fu LL, Liu DM, Liu HQ and Mei CL: VEGF-modified human embryonic mesenchymal stem cell implantation enhances protection against cisplatin-induced acute kidney injury. Am J Physiol Renal Physiol 300: F207-F218, 2011.

101. Regoli D, Plante GE and Gobeil F Jr: Impact of kinins in the treatment of cardiovascular diseases. Pharmacol Ther 135 94-111, 2012.

102. Xiong W, Chen LM, Woodley-Miller C, Simson JA and Chao J: Identification, purification, and localization of tissue kallikrein in rat heart. Biochem J 267: 639-646, 1990.

103. Nolly H, Carbini LA, Scicli G, Carretero OA and Scicli AG: A local kallikrein-kinin system is present in rat hearts. Hypertension 23: 919-923, 1994.

104. Wolf WC, Harley RA, Sluce D, Chao L and Chao J: Localization and expression of tissue kallikrein and kallistatin in human blood vessels. J Histochem Cytochem 47: 221-228, 1999.

105. Agata J, Chao L and Chao J: Kallikrein gene delivery improves cardiac reserve and attenuates remodeling after myocardial infarction. Hypertension 40: 653-659, 2002.

106. Yao YY, Yin H, Shen B, Chao L and Chao J: Tissue kallikrein infusion prevents cardiomyocyte apoptosis, inflammation and ventricular remodeling after myocardial infarction. Regul Pept 140: 12-20, 2007.

107. Yao YY, Yin H, Shen B, Smith RS Jr, Liu Y, Gao L, Chao L and Chao J: Tissue kallikrein promotes neovascularization and improves cardiac function by the Akt-glycogen synthase kinase-3beta pathway. Cardiovasc Res 80: 354-364, 2008.

108. Yin H, Chao L and Chao J: Kallikrein/kinin protects against myocardial apoptosis after ischemia/reperfusion via Akt-glycogen synthase kinase-3 and Akt-Bad.14-3-3 signaling pathways. J Biol Chem 280: 8022-8030, 2005.

109. Westermann D, Schultheiss HP and Tschöpe C: New perspective on the tissue kallikrein-kinin system in myocardial infarction: Role of angiogenesis and cardiac regeneration. Int Immunopharmacol 8: 148-154, 2008.

110. Yin $\mathrm{H}$, Chao L and Chao J: Nitric oxide mediates cardiac protection of tissue kallikrein by reducing inflammation and ventricular remodeling after myocardial ischemia/reperfusion. Life Sci 82: 156-165, 2008

111. Yayama K, Wang C, Chao L and Chao J: Kallikrein gene delivery attenuates hypertension and cardiac hypertrophy and enhances renal function in Goldblatt hypertensive rats. Hypertension 31: 1104-1110, 1998. 
112. Wolf WC, Yoshida H, Agata J, Chao L and Chao J: Human tissue kallikrein gene delivery attenuates hypertension, renal injury, and cardiac remodeling in chronic renal failure. Kidney Int 58: 730-739, 2000.

113. Bledsoe G, Chao L and Chao J: Kallikrein gene delivery attenuates cardiac remodeling and promotes neovascularization in spontaneously hypertensive rats. Am J Physiol Heart Circ Physiol 285: H1479-H1488, 2003.

114. Chao J, Shen B, Gao L, Xia CF, Bledsoe G and Chao L: Tissue kallikrein in cardiovascular, cerebrovascular and renal diseases and skin wound healing. Biol Chem 391: 345-355, 2010.

115. Spillmann F, Graiani G, Van Linthout S, Meloni M, Campesi I, Lagrasta C, Westermann D, Tschöpe C, Quaini F, Emanueli C and Madeddu P: Regional and global protective effects of tissue kallikrein gene delivery to the peri-infarct myocardium. Regen Med 1: 235-254, 2006.

116. Emanueli C, Minasi A, Zacheo A, Chao J, Chao L, Salis MB, Straino S, Tozzi MG, Smith R, Gaspa L, et al: Local delivery of human tissue kallikrein gene accelerates spontaneous angiogenesis in mouse model of hindlimb ischemia. Circulation 103 125-132, 2001.

117. Emanueli C and Madeddu P: Angiogenesis therapy with human tissue kallikrein for the treatment of ischemic diseases. Arch Mal Coeur Vaiss 97: 679-687, 2004.

118. Murakami H, Miao RQ, Chao L and Chao J: Adenovirusmediated kallikrein gene transfer inhibits neointima formation via increased production of nitric oxide in rat artery. Immunopharmacology 44: 137-143, 1999.

119. Murakami H, Yayama K, Miao RQ, Wang C, Chao L and Chao J: Kallikrein gene delivery inhibits vascular smooth muscle cell growth and neointima formation in the rat artery after balloon angioplasty. Hypertension 34: 164-170, 1999.

120. Hagiwara M, Shen B, Chao L and Chao J: Kallikrein-modified mesenchymal stem cell implantation provides enhanced protection against acute ischemic kidney injury by inhibiting apoptosis and inflammation. Hum Gene Ther 19: 807-819, 2008.

121. González A, Ravassa S, Beaumont J, López B and Díez J: New targets to treat the structural remodeling of the myocardium. J Am Coll Cardiol 58: 1833-1843, 2011.

122. Tschöpe C, Walther T, Königer J, Spillmann F, Westermann D Escher F, Pauschinger M, Pesquero JB, Bader M, Schultheiss HP and Noutsias M: Prevention of cardiac fibrosis and left ventricular dysfunction in diabetic cardiomyopathy in rats by transgenic expression of the human tissue kallikrein gene. FASEB J 18 828-835, 2004.

123. Yao Y, Sheng Z, Li Y, Fu C, Ma G, Liu N, Chao J and Chao L: Tissue kallikrein-modified human endothelial progenitor cell implantation improves cardiac function via enhanced activation of akt and increased angiogenesis. Lab Invest 93: 577-591, 2013

124. Naicker S, Naidoo S, Ramsaroop R, Moodley D and Bhoola K: Tissue kallikrein and kinins in renal disease. Immunopharmacology 44: 183-192, 1999.

125. Katori M and Majima M: A missing link between a high salt intake and blood pressure increase. J Pharmacol Sci 100: 370-390, 2006

126. Sharma JN and Narayanan P: The kallikrein-kinin pathways in hypertension and diabetes. Prog Drug Res 69: 15-36, 2014.

127. Uehara Y, Hirawa N, Kawabata Y, Suzuki T, Ohshima N, Oka K, Ikeda T, Goto A, Toyo-oka T and Kizuki K: Long-term infusion of kallikrein attenuates renal injury in Dahl salt-sensitive rats. Hypertension 24: 770-778, 1994.

128. Chao J, Zhang JJ, Lin KF and Chao L: Adenovirus-mediated kallikrein gene delivery reverses salt-induced renal injury in Dahl salt-sensitive rats. Kidney Int 54: 1250-1260, 1998.

129. Hirawa N, Uehara Y, Suzuki T, Kawabata Y, Numabe A, Gomi T, lkeda T, Kizuki K and Omata M: Regression of glomerular injury by kallikrein infusion in Dahl salt-sensitive rats is a bradykinin B2-receptor-mediated event. Nephron 81: 183-193, 1999.
130. Bledsoe G, Shen B, Yao Y,Zhang JJ, Chao L and Chao J: Reversal of renal fibrosis, inflammation, and glomerular hypertrophy by kallikrein gene delivery. Hum Gene Ther 17: 545-555, 2006.

131.Zhang JJ, Bledsoe G, Kato K, Chao L and Chao J: Tissue kallikrein attenuates salt-induced renal fibrosis by inhibition of oxidative stress. Kidney Int 66: 722-732, 2004

132.Liu Y, Bledsoe G, Hagiwara M, Yang ZR, Shen B, Chao L and Chao J: Blockade of endogenous tissue kallikrein aggravates renal injury by enhancing oxidative stress and inhibiting matrix degradation. Am J Physiol Renal Physiol 298: F1033-F1040, 2010.

133.Xia CF, Bledsoe G, Chao L and Chao J: Kallikrein gene transfer reduces renal fibrosis, hypertrophy, and proliferation in DOCA-salt hypertensive rats. Am J Physiol Renal Physiol 289: F622-F631, 2005

134. Schanstra JP, Neau E, Drogoz P, Arevalo Gomez MA, Lopez Novoa JM, Calise D, Pecher C, Bader M, Girolami JP and Bascands JL: In vivo bradykinin B2 receptor activation reduces renal fibrosis. J Clin Invest 110: 371-379, 2002.

135. Bledsoe G, Shen B, Yao YY, Hagiwara M, Mizell B, Teuton M, Grass D, Chao L and Chao J: Role of tissue kallikrein in prevention and recovery of gentamicin-induced renal injury. Toxicol Sci 102: 433-443, 2008

136. Dellalibera-Joviliano R, Reis ML and Donadi EA: Kinin system in lupus nephritis. Int Immunopharmacol 1: 1889-1896, 2001.

137.Liu K, Li QZ, Delgado-Vega AM, Abelson AK, Sánchez E, Kelly JA, Li L, Liu Y, Zhou J, Yan M, et al; Profile Study Group; Italian Collaborative Group; German Collaborative Group; Spanish Collaborative Group; Argentinian Collaborative Group; SLEGEN Consortium: Kallikrein genes are associated with lupus and glomerular basement membrane-specific antibody-induced nephritis in mice and humans. J Clin Invest 119: 911-923, 2009.

138. Li Y, Raman I, Du Y, Yan M, Min S, Yang J, Fang X, Li W, Lu J, Zhou XJ, et al: Kallikrein transduced mesenchymal stem cells protect against anti-GBM disease and lupus nephritis by ameliorating inflammation and oxidative stress. PLoS One 8: e67790, 2013.

139. Xia CF, Yin H, Borlongan CV, Chao L and Chao J: Kallikrein gene transfer protects against ischemic stroke by promoting glial cell migration and inhibiting apoptosis. Hypertension 43: 452-459, 2004.

140.Zhang JJ, Chao L, Chao J, Chu Y and Heistad DD: Adenovirus-mediated kallikrein gene delivery reduces aortic thickening and stroke-induced death rate in Dahl salt-sensitive rats. Stroke 30: 1925-1931, discussion 1931-1932, 1999.

141.Xia CF, Yin H, Yao YY, Borlongan CV, Chao L and Chao J: Kallikrein protects against ischemic stroke by inhibiting apoptosis and inflammation and promoting angiogenesis and neurogenesis. Hum Gene Ther 17: 206-219, 2006.

142. Chao J and Chao L: Experimental therapy with tissue kallikrein against cerebral ischemia. Front Biosci 11: 1323-1327, 2006.

143. Kizuki K, Iwadate H and Ookubo R: Growth-stimulating effect of kallikrein on rat neural stem cells - II. Immunocytochemical analysis and specificity of the enzyme for neural stem cells. Yakugaku Zasshi 127: 919-922, 2007.

144.Liu L, Liu H, Yang F, Chen G, Zhou H, Tang M, Zhang R and Dong Q: Tissue kallikrein protects cortical neurons against hypoxia/reoxygenation injury via the ERK1/2 pathway. Biochem Biophys Res Commun 407: 283-287, 2011.

This work is licensed under a Creative Commons Attribution-NonCommercial-NoDerivatives 4.0 International (CC BY-NC-ND 4.0) License. 